\title{
Implementation and Evaluation of Infertility Reflection in Early Pregnancy after the Use of Assisted Reproductive Technology: A Feasibility Study
}

\author{
Takayo Sakiyama \\ Department of Nursing, Ehime University Graduate of Medicine, Toon, Japan \\ Email: sakiyama.takayo.xk@ehime-u.ac.jp
}

How to cite this paper: Sakiyama, $\mathrm{T}$. (2021) Implementation and Evaluation of Infertility Reflection in Early Pregnancy after the Use of Assisted Reproductive Technology: A Feasibility Study. Open Journal of Nursing, 11, 477-488.

https://doi.org/10.4236/ojn.2021.116041

Received: April 27, 2021

Accepted: June 21, 2021

Published: June 24, 2021

Copyright $\odot 2021$ by author(s) and Scientific Research Publishing Inc. This work is licensed under the Creative Commons Attribution International License (CC BY 4.0).

http://creativecommons.org/licenses/by/4.0/

\begin{abstract}
Background: This study aims to evaluate the infertility reflection in early pregnancy after assistive reproductive therapy (ART), including 1) process evaluation (the use and evaluation of infertility reflection) and 2) outcome evaluation (satisfaction of care needs, anticipatory anxiety towards the loss of a pregnancy or fetus, cognition of infertility experience, and depression and anxiety). Methods: This program evaluation study used a one-group pre-post-test design. The participants were 50 primiparas who had undergone ART at two fertility treatment facilities in a metropolitan area in Japan. For the infertility reflection, they conducted an online reflection. Data were collected three times: at the 5th week of pregnancy (Time 1), the 8th week of pregnancy as the final consultation at the clinic (Time 2), and the 16th week of pregnancy as the final point of early pregnancy (Time 3 ). Results: The data from 40 participants were analyzed. More than $80 \%$ of the users of the online reflection positively evaluated the appropriateness and usefulness of the methods and contents. Organized thoughts and feelings by reflection were shown as the reasons for the usefulness. The evaluation of the online reflection showed a relatively strong correlation with the Care Need Satisfaction Scale (CNSS) for both Time 2 and Time 3, but the online reflection did not show a significant correlation with the other outcome variables. There were no significant differences in outcome variables between users and non-users of online reflection between Time 2 and Time 3. Conclusions: Attempts at the reflection in early pregnancy require modified methods that do not have a negative impact and lead to the fulfillment of needs.
\end{abstract}

\section{Keywords}

Infertility Reflection, Online Support Program, Assisted Reproductive 
Technology, Early Pregnancy, Program Evaluation

\section{Introduction}

Reproduction is a key developmental task in adulthood; infertility is a crisis. The stress and disappointment experienced by women who suffer from infertility have negative impacts on their self-esteem, mental health, and marital relationships [1] [2] [3]. Pregnancy after undergoing fertility treatment is the process of transition from the state of infertility to becoming a mother, and many women in this situation are worried about returning to infertility after a miscarriage [4]. Such women engage in psychological preparation in order to minimize the shock that is associated with a miscarriage, even as they regard their pregnancy as yet unconfirmed and often have only inadequate information about the condition [5] [6] [7]. The effort that is needed to overcome this uncertainty is special and different from that required for a natural pregnancy. In addition, if the act of looking back on the infertility experience during treatment is incomplete, it tends to be difficult to adapt to pregnancy, childbirth, and childcare. Therefore, it is necessary to reflect on the infertility experience [5].

Although the mental health of pregnant women tends to stabilize in the second trimester, women who have used assistive reproductive therapy (ART) have high anxiety about miscarriage and maternal-fetal abnormalities during early pregnancy. These anxieties show a significant correlation with avoidance feelings toward the fetus in the third trimester [8]. Furthermore, the apprehensions regarding a miscarriage correlate with a delay in the preparation for the maternal role and a decline in maternal self-efficacy [9] [10]. In addition, anticipatory anxiety about loss is highly evident when the infertility experience is not taken positively. Depression and trait anxiety are intensely manifest when the infertility experience is viewed negatively. This condition indicates that mental health in early pregnancy is adversely affected by the infertility experience [11]. Women in early pregnancy have care needs for emotional support, guidance, infertility reflection, and peer sharing [12]. The infertility reflection, one of the care needs, showed expectations for expressing and reconstructing experiences and feelings during fertility treatment. Infertility reflection is consistent with the work required to transition from infertility to becoming a mother. However, only $10 \%$ or less of the facilities in Japan provide care that help solve conflicts and worries through infertility reflections [13]. The reason is that the effects of infertility reflection have not yet been verified.

The objective of this study is to evaluate the infertility reflection in early pregnancy after undergoing ART, including 1) process evaluation (the use and evaluation of infertility reflection) and 2) outcome evaluation (satisfaction of care needs, anticipatory anxiety toward the loss of a pregnancy or fetus, cognition of infertility experience, and depression and anxiety). 


\section{Methods}

\subsection{Design}

This program evaluation study used a one-group pre-post-test design.

\subsection{Participants}

The participant sample consisted of 50 primiparas who had undergone ART at two fertility treatment facilities in a metropolitan area in Japan. The study exclusion criteria were as follows: 1) gamete provision by a donor, and 2) mental illness. Both facilities had certified nurses for infertility nursing and clinical psychologists, and support during treatment was provided to the same extent.

\subsection{Intervention Contents}

\subsubsection{Intervention Methods}

The infertility reflection aimed at allowing pregnant women who had undergone ART to evaluate their infertility experience as a positive experience. For the infertility reflection, they conducted an online reflection using a reflection sheet. These methods were created based on the care needs of early pregnancy nursing support programs [12].

The reflection sheet comprised descriptions of 1 ) the infertility experience; 2) resetting goals; 3) the prediction of available support; and 4) feedback on the reflection, based on the concept of pregnancy adaptation [14]. The contents of the reflection sheet are listed in Table 1 . The validity of the contents and the method of the sheet were confirmed by certified nurses and clinical psychologists at research cooperation facilities, and women who had experienced pregnancy and childbirth by ART. Points 1), 2), and 3) were written by subjects on the sheet, and 4) the feedback on the reflection sheets they submitted was given by the researcher. The feedback perspective was based on the following aspects: 1) showing empathy; 2) praising their efforts; 3 ) correcting any misinterpretation of facts; 4) asking the reason for and encouraging the change in thinking if negative feelings alone were expressed; 5) recommending the help of certified nurses or clinical psychologists if their concerns required counseling; and 6) not forcing a reflection if they indicated that they seemed disinclined.

\subsubsection{Intervention Protocol}

The infertility reflection is part of the Adaptation Support Program in early pregnancy [15]. The gestational sac was diagnosed at the 5th week of pregnancy. We had explained that the infertility reflection was one element of the Adaptation Support Program, and cooperation was requested. We had also demonstrated the use of the online reflection with a computer and had given the women their login IDs. The reflection became available one week after the explanation. If the reflection sheet was written and sent, the researcher checked the sheet and provided the women with a feedback. The intervention period was approximately two weeks before the transfer. 
Table 1. Contents of the reflect sheet.

Introduction

How did you experience infertility treatment?

As soon as you found out you were pregnant, you may have revived various thoughts.

From now on you will proceed to pregnancy step.

Let's organize the thought until now, and imagine the future.

Description items

1. Let's write the feelings of the present.

2. What did you think of the infertility experience? Let's look back on both the good and bad sides.

3. How did you solve the troubles concerning infertility?

(Example: I consulted my partner, gathering information, I could not talk to anyone, etc.)

4. What kind of pregnancy life do you want?

(Example: spend so as not to put a burden on the baby, work on handicrafts for babies, etc.)

5. What kind of childbirth or childcare do you want? How is it likely to lead to the determination of the birth place? Let's refer to the content of online resources.

6. How will you solve your future troubles and worries in order to live a desired pregnancy life?

(Example: Consult with a partner, because I could not consult well during treatment, I gradually increase counselors, gathering information, etc.)

7. Let's write it if you have any concerns or problems that you cannot resolve.

\subsection{Data Collection}

Data were collected three times: the 5th week of pregnancy (Time 1), the 8th week of pregnancy as the final consultation at the clinic (Time 2), and the 16th week of pregnancy as the final point of early pregnancy (Time 3 ). At Time 1, the questionnaire was distributed in person after obtaining informed consent for participation from all the respondents, and collected after it was filled out. At Times 2 and 3, it was distributed by mail and then collected.

\subsection{Measures and Measurement Instruments}

The participants' age, pregnancy experience, miscarriage experience, infertility period, fertility treatment period, infertility cause, treatment method, number of embryo transfers, and progress of pregnancy were collected from the medical records at Time 1. In the process evaluation, measurement tool 1 was used for infertility reflection, and tool 2 was used to evaluate the reflection. In the outcome evaluation, tool 3 was used for satisfaction with care needs, and tool 4 was used as anticipatory anxiety toward the loss. Tools 5 and 6 were used in cognition of the infertility experience. Tool 7 was used for depression, and tool 8 was used for anxiety. Tool 1 and tool 2 were used at Time 2, and tool 3 was used at Time 2 and Time 3 . Tools 3 - 8 were used for all the periods.

\subsubsection{Use of the Online Reflection}

The use of the online reflection was confirmed.

\subsubsection{Evaluation of the Online Reflection}

This scale consisted of four items: "appropriateness of methods," "usefulness," "appropriateness of presentation time," and "appropriateness of contents." A 
5-point scale was used; higher scores indicated better evaluations. In addition, the participants were asked to provide their opinion of the infertility reflection through free description.

\subsubsection{Care Need Satisfaction Scale}

The Care Need Satisfaction Scale (CNSS) consists of four factors that are made up of 21 items. It includes questions regarding the "satisfaction of the need to review infertility experience and pregnancy," "satisfaction of the need to share and reduce anxiety and worry," "satisfaction of the need to connect with a peer," and "satisfaction of the need to take on role behavior related to birth and childcare." A 5-point scale was again used, and higher scores indicated higher care need satisfaction. The Cronbach's $\alpha$ of the whole measure was 0.92 , and that of the subscales ranged from 0.75 to 0.87 .

\subsubsection{Anticipatory Anxiety for Loss Scale}

The Anticipatory Anxiety for Loss Scale (AALS) consists of two factors that are made up of 10 items. It includes questions on the "stagnation of joy over the pregnancy" and "worry about the loss of a normal pregnancy." A 7-point scale was used, and higher scores indicated higher anticipatory anxiety regarding the loss of the pregnancy. The Cronbach's $\alpha$ of the whole measure was 0.76 , and that of the subscales ranged from 0.76 to 0.89 . The correlation coefficient of measurement tool 8 was 0.46 . The criterion validity was confirmed because the correlation coefficient with the score of the Hospital Anxiety and Depression Scale-Japanese (HADS-J) was 0.46 .

\subsubsection{Post-Traumatic Growth Inventory-Japanese}

The Post-traumatic Growth Inventory-Japanese (PTGI-J) measures growth after a critical event, and its reliability and validity have been established [16]. It consists of four factors that are made up of 18 items, which include questions on the "relationship with others," "new possibilities," "strength as a human being", and "appreciation for spiritual modification and life." A 6-point scale was used, and higher scores indicated greater growth through the infertility experience.

\subsubsection{Negative Modification Scale}

The Negative Modification Scale (NMS) consists of one factor with these four items: "I found it hard to trust others," "I have lost a lot of things," "I feel blame and disgust toward myself", and "I have lamented my life." A 6-point scale was used, and higher scores indicated more negative modification due to the infertility experience. The Cronbach's $\alpha$ was 0.78 .

\subsubsection{Edinburgh Postnatal Depression Scale}

The Edinburgh Postnatal Depression Scale (EPDS) measures postnatal depression, as discussed in the previous section [17]. This scale consists of 10 items. A 4-point scale was used; higher scores indicated more severe depressive symptoms, and a score of 9 or higher was considered as the depressive group. As its 
use in pregnancy is under consideration, its criterion-related validity was confirmed against "depression" on the HADS-J, the reliability and validity of which have been established [18]. The resulting correlation coefficient was 0.38 .

\subsubsection{State-Trait Anxiety Inventory-Japanese}

The State-Trait Anxiety Inventory-Japanese (STAI-J) measures anxiety. Its reliability and validity have been established [19]. It consists of two factors made up of 20 items: "state anxiety" and "trait anxiety." Only trait anxiety was used here (as state anxiety is covered in measurement tool 5 above), on a 4-point scale, and higher scores indicated higher trait anxiety.

\subsection{Procedure}

The data collection was carried out between August 2014 and March 2015 at three time points. In the two cooperating facilities, convenience sampling was carried out by a researcher through the mediation of the doctors or nurse managers at 5 weeks of pregnancy (after detection of the gestational sac).

\subsection{Data Analysis}

Some analyses were carried out using IBM SPSS for Windows v. $22.0 \mathrm{~J}$ (SPSS, Tokyo, Japan). Measurement tools 2 - 8 were analyzed using the correlation analysis of a Kendall. The use of the online reflection and measurement tools 2 8 was analyzed with the Mann-Whitney $\mathrm{U}$ test and $\chi^{2}$ test.

\subsection{Ethical Considerations}

The following points were explained orally to each participant, and written documents were also handed out: the purpose of the study, respect of free will in terms of study participation and withdrawal, compliance with confidentiality obligations by the researcher, and avoiding disadvantage to the participants in cases of miscarriage or non-cooperation with the study. The consent form was signed by all the participants. The study was approved by the relevant ethical review board at St. Luke's International University, Tokyo, Japan (Approval no. 14-044). The study was conducted in accordance with the Declaration of Helsinki and all its amendments.

\section{Results}

Sixty women were asked to cooperate in the study, and 57 agreed $(95.0 \%$ response rate). The dropout rate was $29.8 \%$ and the dropout rate, excluding an early miscarriage, was $19.2 \%$. The data from 40 participants were included in the final analysis and analyzed. The mean age of the participants was $36.0 \pm 4.2$ years, the mean infertility period was $4.6 \pm 2.2$ years, the mean treatment period was $2.3 \pm 1.5$ years, and the mean number of embryo transfers was $2.1 \pm 2.6$. Furthermore, $35.0 \%$ of the participants had a previous pregnancy experience, and $27.5 \%$ had a previous miscarriage experience. 


\subsection{Process Evaluation}

\subsubsection{Use of Online Reflection}

The use of the online reflection was $40.0 \%$. The online reflection and demographics were analyzed using the Mann-Whitney $U$ test and $\chi^{2}$ test, but no significant differences were observed. On the other hand, the Edinburgh Postnatal Depression Scale (EPDS) of reflection users was significantly higher at Time 1 $(\mathrm{U}=103.50, \mathrm{p}=0.025)$.

\subsubsection{Evaluation of Online Reflection}

Seventy-five percent of the participants evaluated the online reflection positively, in terms of "appropriateness of methods," and responded "I think so" or "I think a little"; $70.0 \%$ evaluated its "appropriateness of presentation time," "appropriateness of contents" positively (Figure 1). When limited to reflection users, 81.3\% positively evaluated the "appropriateness of methods," "usefulness," and "appropriateness of contents"; 75.0\% evaluated "appropriateness of presentation time" positively (Figure 2).

Regarding opinions of the online reflection, five participants said: "Online reflection helped me organize my thoughts and feelings." One participant stated thus: "I was unable to do online reflection." Two non-users felt this way: "It is necessary to take a little more time to reflect on the infertility experience."

\subsection{Outcome Evaluation}

\subsubsection{Correlation of the Evaluation of Online Reflection and the Care Need Satisfaction Scale}

The evaluation of the reflection showed a relatively strong correlation with the CNSS for both Time $2(\mathrm{r}=0.51, \mathrm{p}<0.01)$ and Time $3(\mathrm{r}=0.48, \mathrm{p}<0.01)$ (Table 2).

\subsubsection{Correlation of the Evaluation of Online Reflection and the Other Measurement Variables}

The online reflection did not show significant correlation with the other measurement variables.

Appropriateness of presentation time

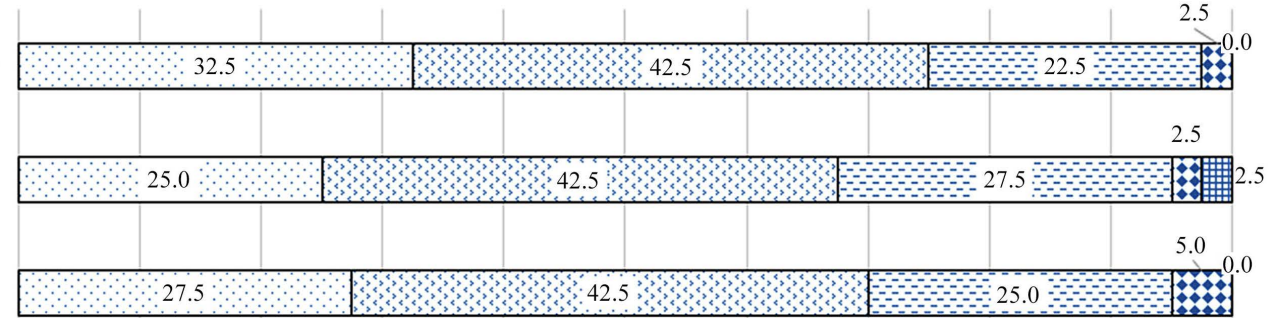

Appr opriateness of contents
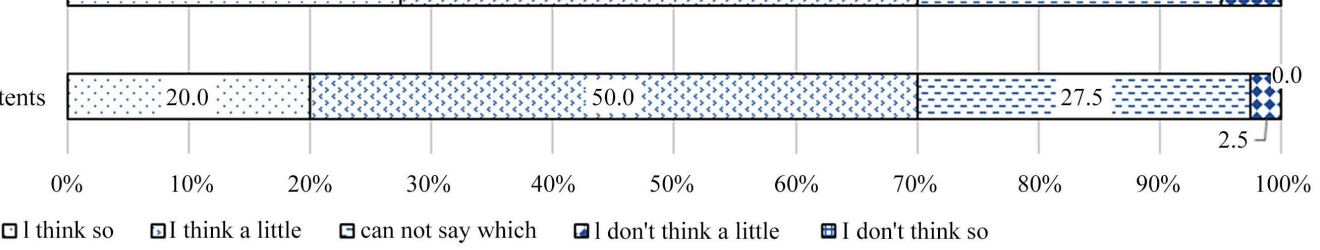

Figure 1. Impression of online reflection. 


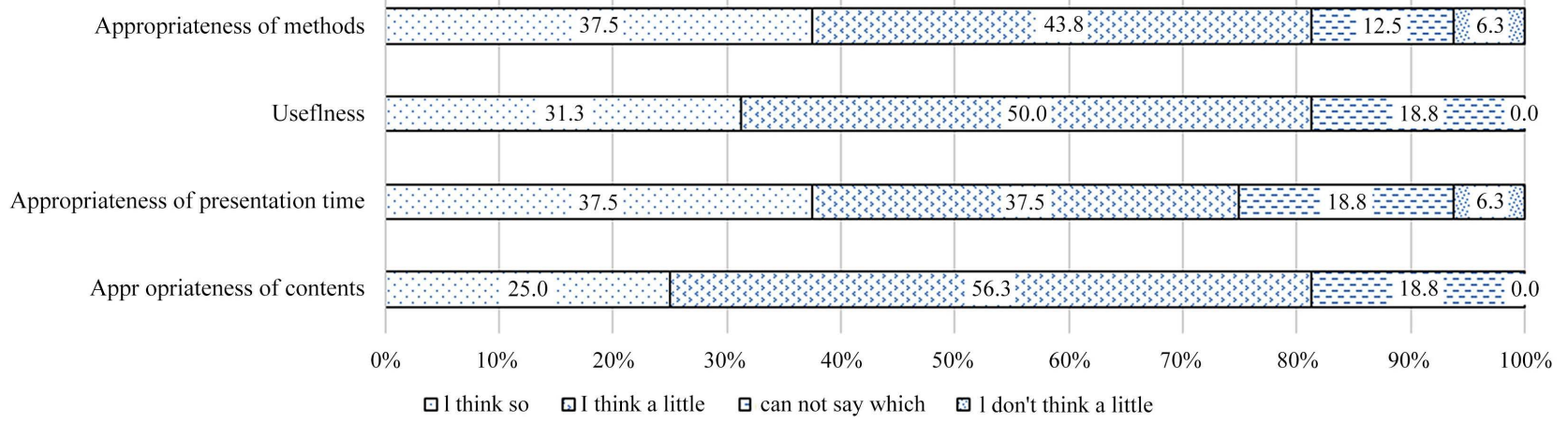

Figure 2. Impression by the user online reflection.

Table 2. Correlations of the evaluation of online reflection and the care need satisfaction scale $(\mathrm{N}=40)$.

\begin{tabular}{|c|c|c|c|c|}
\hline \multirow{3}{*}{ Outcome variables } & \multicolumn{4}{|c|}{ Online reflection } \\
\hline & \multicolumn{2}{|c|}{ Time 2} & \multicolumn{2}{|c|}{ Time 3} \\
\hline & $\mathrm{r}$ & $\mathrm{p}$ & $\mathrm{r}$ & $\mathrm{p}$ \\
\hline Care need satisfaction scale & 0.51 & $<0.01$ & 0.48 & $<0.01$ \\
\hline Satisfaction of need to reflection infertility experience and pregnancy & 0.41 & $<0.01$ & 0.40 & $<0.01$ \\
\hline Satisfaction of need to share and reduce anxiety and worry & 0.43 & $<0.01$ & 0.46 & $<0.01$ \\
\hline Satisfaction of need to have connection with a peer & 0.38 & $<0.01$ & 0.32 & $<0.01$ \\
\hline Satisfaction of need to take on role behavior related to birth and childcare & 0.35 & $<0.01$ & 0.38 & $<0.01$ \\
\hline Anticipatory anxiety for loss scale & -0.20 & 0.07 & -0.22 & 0.06 \\
\hline Post-traumatic growth inventory-Japanese & 0.17 & 0.14 & 0.19 & 0.11 \\
\hline Negative modification scale & 0.14 & 0.24 & 0.02 & 0.82 \\
\hline Edinburgh postnatal depression scale & 0.09 & 0.44 & 0.01 & 0.87 \\
\hline State-trait anxiety inventory-Japanese & -0.02 & 0.82 & -0.14 & 0.22 \\
\hline
\end{tabular}

Kendall's correlation analysis. Time $2=8$ th week of pregnancy, Time $3=16$ th week of pregnancy.

\subsubsection{Comparison between the User and Non-User of Online Reflection the Outcome Variables}

There were no significant differences in outcome variables between users and non-users of online reflection at Time 2 and Time 3.

\section{Discussion}

\subsection{Process Evaluation in Infertility Reflection}

The average of participants' age, infertility treatment period, pregnancy experience, miscarriage experience, and miscarriage rate were consistent with previous studies. This intervention was used by applicants, and the utilization rate was as low as $40 \%$. No characteristics were found in the attributes of the users, and it was inferred that the need for reflection had individuality. The user's depression score was significantly higher before the intervention but did not significantly decrease after the reflection. In other words, this intervention had no effect on reducing depression. More than $70 \%$ of users positively evaluated the appropriateness of the methods, the presentation time, and the content, but the usefulness was slightly low at $67.5 \%$. Non-users registered few negative opinions. 
On the other hand, more than $80 \%$ of users positively evaluated the appropriateness of the methods, contents, and usefulness. The reason for the utility is the feasibility to organize thoughts and feelings through reflection. Pregnancy adaptation after ART corresponds to the developmental transition of becoming a mother. It also corresponds to the transition of the healthcare system, which is transferred from a fertility treatment facility to a delivery facility. Transition is completed when a person recognizes that a safer period has been reached. Therefore, the stress, recovery, and timing of completion of this transition are individual [20]. The PTGI-J of women at the 5th and 8th weeks of pregnancy after they underwent ART showed a negative correlation with AALS [11]. This fact meant that it was difficult to capture the infertility experience positively when there was strong anticipatory anxiety regarding loss. At the 16th week of pregnancy, the correlation disappeared [11]. From these findings, it may be inferred that the number of weeks of pregnancy influences the infertility reflection. However, since there are individual differences in reflection depending on taste, it is important not to force reflection at a specific time and to respect the wishes of women.

\subsection{Outcome Evaluation in Infertility Reflection}

An evaluation of the online reflection showed a correlation with the CNSS at Time 2 and Time 3. In other words, the higher the evaluation of the reflection was, the more satisfied were the care needs. However, reflection users did not show significant differences in all outcome variables compared to non-users. This aspect meant that the care needs were satisfied but had no significant impact on the outcome variables. The goal of the reflection was to evaluate the infertility experience as a positive experience, but it did not show a significant increase in the PTGI-J or a significant decrease in the NMS. This situation may be due to the fact that it was difficult to grasp the infertility experience positively when there was strong anticipatory anxiety for loss at Time 2 and because only the applicants used the reflection in this study. From these findings, it was inferred that the outcome variables were averaged, and no significant difference was observed. It is also possible that the results were influenced by the fact that the researcher was not a staff member at the fertility treatment facility. It is necessary to find ways to better satisfy the care needs of users and to change the outcome variables. Therefore, it is necessary to consider the timing, methods, and practitioners of intervention.

\subsection{Evaluation and Task of Infertility Reflection in Early Pregnancy}

Attempts at reflection in early pregnancy show that the methods need to be modified, but they do not have a negative impact, and lead to the fulfillment of needs. Nurses tend to hesitate to support women during early pregnancy because pregnancy after the use of fertility treatment is more likely to result in a miscarriage [21]. The results of this study may dispel that impression and sup- 
port the promotion of more appropriate nursing practices.

Infertility reflection is premised on respecting the wishes of women, but it is necessary to consider the timing, methods, and practitioners of intervention.

\subsection{Limitations of the Study}

This study aimed at program evaluation, hence it is not an effect study, and the sample size was small. In addition, the researcher was not a staff member of the fertility treatment facility. In future, it will be necessary to increase the number of subjects, modify the intervention method, and verify the effect.

\section{Conclusions}

More than $80 \%$ of the users of online reflection positively evaluated the appropriateness of the methods, contents, and usefulness. The reason for its expediency was attributed to organized thoughts and feelings by means of reflection.

The evaluation of the online reflection showed a relatively strong correlation with the CNSS for both Time 2 and Time 3, but the online reflection did not show a significant correlation with the other outcome variables. In addition, there were no significant differences in outcome variables between users and non-users of online reflection at Time 2 and Time 3. From the results mentioned above, it is clear that attempts at the reflection in early pregnancy need modified methods, though they do not have a negative impact and lead to the fulfillment of needs. Infertility reflection is premised on respecting the wishes of women, but it is necessary to consider the timing, methods, and practitioners of intervention.

\section{Acknowledgements}

The author would like to thank all the participating women in this study and the facility staff members. This research was conducted for the author's doctoral dissertation at St. Luke's International University, Tokyo, Japan. The author deeply appreciates the valuable advice provided by Professor Akiko Mori. This study was supported by the Fumiko Yamaji Nursing Research Fund and the Policy-Based Medical Services Foundation, Japan.

\section{Conflicts of Interest}

The author declares no conflict of interest.

\section{Author' Contributions}

T.S. conceived and designed the study, acquired and analyzed the data, drafted and revised the manuscript, and approved the final version of the manuscript.

\section{References}

[1] Gameiro, S., Moura-Ramos, M., Canavarro, M.C. and Soares, I. (2010) Psychosocial Adjustment during the Transition to Parenthood of Portuguese Couples who Con- 
ceived Spontaneously or through Assisted Reproductive Technologies. Research in Nursing \& Health, 33, 207-220. https://doi.org/10.1002/nur.20377

[2] Olshansky, E.F. and Sereika, S. (2005) The Transition from Pregnancy to Postpartum in Previously Infertile Women: A Focus on Depression. Archives of Psychiatric Nursing, 19, 273-280. https://doi.org/10.1016/j.apnu.2005.08.003

[3] Brennan, D.P., Camilla, S.S., Matthew, P. and Lone, S. (2014) Are Severe Depressive Symptoms Associated with Infertility-Related Distress in Individuals and Their Partners? Human Reproduction, 29, 76-82. https://doi.org/10.1093/humrep/det412

[4] Sandelowski, M. (1995) A Theory of the Transition to Parenthood of Infertile Couples. Research in Nursing \& Health, 18, 123-132.

https://doi.org/10.1002/nur.4770180206

[5] Bernstein, J. (1990) Parenting after Infertility. The Journal of Perinatal and Neonatal Nursing, 4, 11-23. https://doi.org/10.1097/00005237-199009000-00004

[6] Covington, S.N. and Burns, L.H. (2006) Pregnancy after Infertility. In: Covington, S.N. and Burns, L.H., Eds., Infertility Counseling: A Comprehensive Handbook for Clinicians, 2nd Edition, Cambridge University Press, Cambridge, 440-458. https://doi.org/10.1017/CBO9780511547263.027

[7] Mori, E., Ishii, K. and Hayashi, H. (2007) Prenatal Maternal Role Attainment after Infertility Treatment. Journal of Japanese Society of Fertility Nursing, 4, 26-33. (In Japanese)

[8] Mori, E., Chen, D. and Nukazuka, A. (2005) The Effect of Experiences of Infertility and Infertility Treatment on Maternal Feeling. Journal of Japanese Society of Fertility Nursing, 2, 28-35. (In Japanese)

[9] McMahon, C.A., Ungerer, J.A., Tennant, C. and Saunders, D. (1997) Psychosocial Adjustment and the Quality of the Mother-Child Relationship at Four Months Postpartum after Conception by in Vitro Fertilization. Fertility and Sterility, 68, 492-500. https://doi.org/10.1016/S0015-0282(97)00230-6

[10] Maehara, K., Mori, E., Ozawa, H., Maekawa, T., Morita, A., Iwata, H., et al. (2012) Relation of Maternal Anxiety to Feelings toward the Infant and Maternal Role Attainment in Pregnant Women after Assisted Reproductive Technology. Journal of Graduate School of Nursing, 34, 1-8. (In Japanese)

[11] Sakiyama, T. (2020) Relationships among Perception of Infertility Experience, Anxiety, and Depression in Early Pregnancy after the Use of Assisted Reproductive Technology. Journal of Reproductive Psychology, 6, 54-60. (In Japanese)

[12] Sakiyama, T. (2015) Women's Needs Focused on Nursing Care in Early Pregnancy after Undergoing Assisted Reproductive Technology. Japanese Journal of Maternal Health, 56, 349-358. (In Japanese)

[13] Sakiyama, T. (2015) Factors Influencing Early Pregnancy Nursing Care at Japanese Fertility Treatment Facilities by Covariance Structure Analysis. Journal of Japanese Society of Fertility Nursing, 12, 15-23. (In Japanese)

[14] Sakiyama, T. (2013) Pregnancy Adaptation by Japanese Women Pregnant by Means of Fertility Treatment: A Concept Analysis. Journal of Japanese Society of Fertility Nursing, 10, 51-57. (In Japanese)

[15] Sakiyama, T. (2019) Development and Evaluation of the Adaptation Support Program in Early Pregnancy after the Use of Assisted Reproductive Technology. Japan Journal of Nursing Science, 16, 286-299. https://doi.org/10.1111/jins.12238

[16] Taku, K. (2005) Study on the Mechanism of Sense of Self Growth Caused by Stress. Journal of Japanese Clinical Psychology, 23, 161-172. (In Japanese) 
[17] Okano, T., Murata, M., Masuji, A., Tamaki, R., Nomura, J., Miyaoka, H., et al. (1996) Reliability and Validity of EPDS in Japan. Archives of Psychiatric Diagnostics and Clinical Evaluation, 7, 523-533. (In Japanese)

[18] Zigmond, A.S., Snaith, R.P. and Kitamura, T. (1983) Hospital Anxiety and Depression Scale. Archives of Psychiatric Diagnostics and Clinical Evaluation, 4, 371-372.

[19] Hidano, T., Hukuhara, M., Iwawaki, S., Soga, S. and Spielberger, C. (2000) State-Trait Anxiety Inventoryform. Jitsumukyoiku-Shuppan Company, Tokyo. (In Japanese)

[20] Chick, N. and Meleis, A.I. (1986) Transition: A Nursing Concern. In: Chinn, P.L., Ed., Nursing Research Methodology, Aspen Publication, Boulder, CO, 237-257. http://repository.upenn.edu/nrs

[21] Sakiyama, T. (2016) Analysis of the Nursing Services Provided for Early Pregnancy at Japanese Fertility Treatment Centers. Japanese Journal of Maternal Health, 56, 634-642. (In Japanese) 